\title{
Integration of additive manufacturing and inkjet printed electronics: a potential route to parts with embedded multifunctionality
}

\author{
Jonathan Stringer ${ }^{1, *}$, Talal M. Althagathi ${ }^{3}$, Christopher C.W. Tse ${ }^{2}$, Van Duong Ta ${ }^{4}$, \\ Jonathan D. Shephard ${ }^{4}$, Emre Esenturk ${ }^{5}$, Colm Connaughton ${ }^{5,6}$, Thomas J. Wasley ${ }^{7}, \mathrm{Ji} \mathrm{Li}^{7}$, \\ Robert W. Kay ${ }^{7}$, and Patrick J. Smith ${ }^{2}$ \\ 1 Department of Mechanical Engineering, University of Auckland, 20 Symonds St., Auckland, New Zealand \\ 2 Laboratory of Applied Inkjet Printing, Department of Mechanical Engineering, Garden Street, University of Sheffield, \\ Sheffield S1 4BJ, UK \\ 3 Department of Physics and Astronomy, University of Sheffield, Hicks Building, Hounsfield Rd, Sheffield S3 7RH, UK \\ 4 Institute of Photonics and Quantum Sciences, Heriot-Watt University, Edinburgh, EH14 4AS, UK \\ 5 Warwick Mathematics Institute, Zeeman Building, University of Warwick, Coventry CV4 7AL, UK \\ ${ }^{6}$ Centre for Complexity Science, Zeeman Building, University of Warwick, Coventry CV4 7AL, UK \\ 7 Additive Manufacturing Research Group, Loughborough University, Leicestershire LE11 3TU, UK
}

Received 25 January 2016 / Accepted 11 April 2016

\begin{abstract}
Additive manufacturing, an umbrella term for a number of different manufacturing techniques, has attracted increasing interest recently for a number of reasons, such as the facile customisation of parts, reduced time to manufacture from initial design, and possibilities in distributed manufacturing and structural electronics. Inkjet printing is an additive manufacturing technique that is readily integrated with other manufacturing processes, eminently scalable and used extensively in printed electronics. It therefore presents itself as a good candidate for integration with other additive manufacturing techniques to enable the creation of parts with embedded electronics in a timely and cost effective manner. This review introduces some of the fundamental principles of inkjet printing; such as droplet generation, deposition, phase change and post-deposition processing. Particular focus is given to materials most relevant to incorporating structural electronics and how post-processing of these materials has been able to maintain compatibility with temperature sensitive substrates. Specific obstacles likely to be encountered in such an integration and potential strategies to address them will also be discussed.
\end{abstract}

Key words: Inkjet Printing, Additive Manufacturing, Printed Electronics

\section{Introduction}

In recent years there has been increasing interest in additive manufacturing, manufacturing processes that translate information from a three dimensional data file to selectively form a part from a feedstock in a layer-by-layer fashion [1]. As an object manufactured in this way is based solely on a data file, it is a trivial process to alter or customise the design depending upon the specific application. This has resulted in a great deal of interest in areas where facile customisation would be desirable, for example in orthotics and prosthesis [2]. In comparison to more traditional manufacturing techniques, additive manufacturing also presents advantages in being able to achieve complex internal geometries [3], and enables the use of designs that would be uneconomic to manufacture despite potentially

*Corresponding author: j.stringer@auckland.ac.nz being more desirable from a purely engineering [4] or aesthetic perspective [5]. The information for the part being solely represented by the data file also presents possibilities in distributed manufacturing [6].

The roots of additive manufacturing can be traced back to a series of similar technologies developed in the 1980s that were used for rapid visualisation of designs and prototypes, and was therefore referred to as rapid prototyping [7]. As the primary aims of the technology at this point were to physically replicate a given 3D data file for visualisation, little attention was paid to the mechanical properties of the produced part or the materials used within the part. Over time, the potential for the rapid prototyping techniques to be used as a means of manufacturing was identified, which lead to further research into the use of more industrially relevant materials, particularly metals, in additive manufacturing processes [8]. 
Additive manufacturing is an umbrella term that covers a number of different technologies that all follow the basic definition provided above. While the focus of this review is not on additive manufacturing itself (the authors refer the reader to more in-depth reviews such as those by Wong and Hernandez [9] and Guo and Leu [10]) a brief summary of the different additive manufacturing techniques follows.

The current technologies for additive manufacturing can be loosely divided into four categories, namely direct material deposition, sheet lamination, powder bed fusion and selective photopolymerisation. The category of direct material deposition includes technologies whereby material is extruded where needed (e.g. fused deposition modelling and robocasting), where it is printed as droplets (such as inkjet printing) or where the material as a heated powder feedstock is dispensed where required (e.g. laser engineered net shaping and direct materials deposition). The material is deposited in a liquid state (either molten, in solution, suspension or slurry or as an uncured resin) and subsequently changes phase in the desired location. The nature of this phase change, and the prerequisites required to obtain the initial liquid state vary depending upon the material used, with metallic and ceramic materials needing a higher initial temperature (provided by an electron beam or laser). The resolution capable with these techniques is dependent upon size of the orifice through which the material is extruded, with the final resolution typically being the same size or slightly larger. For inkjet printing, the orifice diameter lies within the range of 10-100 $\mu \mathrm{m}$, while for robocasting the orifice diameter is typically $100-1000 \mu \mathrm{m}$. Techniques similar in principle to robocasting have demonstrated a resolution far finer than this (e.g. $6 \mu \mathrm{m}$ [11] and $1 \mu \mathrm{m}$ [12]), although it should be noted that this increase in resolution gives a consequential increase in deposition time for a given volume of material.

Sheet lamination techniques include ultrasonic consolidation and laminated object manufacturing. They function by cutting each required layer followed by bonding to previously bonded layers until the final object is complete. This bonding is either via an adhesive in the case of laminated object manufacturing, or as a solid state weld for ultrasonic consolidation. Powder bed fusion, as the name would suggest, relies on the selective fusion of powder particles within a powder bed, followed by the now fused layers being recoated with further powder and the process repeated until the part is manufactured. The nature of how the powder is fused together is either the selective application of sufficient energy to sinter or melt the powder (typically by a laser or electron beam), or the use of an adhesive binder that penetrates the powder bed by capillary action. The size of the powder particles within the powder bed is the fundamental limitation on the achievable resolution in powder bed processes, and for a component to have adequate strength and surface finish it is typical that a feature must be several times the size of a single powder particle. The size of the powder is primarily limited by ease of handling during processing, with finer powders presenting problems with the build-up of static electricity as well as potential health issues. This, together with a typically increased cost for smaller diameter powders, means that powder diameter is limited to approximately $50 \mu \mathrm{m}$, corresponding to a feature resolution of approximately $0.3-1 \mathrm{~mm}$.
Selective photopolymerisation techniques rely on the use of resin that undergoes crosslinking when subjected to (typically) UV light energy. The resin is subjected to this resin selectively, either by scanning the resin with a UV laser beam or by projecting UV light only where desired. The now cured resin layer is then recoated with further resin and the process repeated until the desired object is fabricated. While only fundamentally limited by the wavelength of incident light, most commercially available stereolithography equipment has an achievable resolution within the range of $10-100 \mu \mathrm{m}$, although micron and sub-micron features are possible with techniques such as 2 photon polymerisation [13] and microstereolithography [14].

While there is clearly great potential for the utilisation of additive manufacturing, there are still currently a number of substantial issues that limit this. Compared to more conventional manufacturing techniques, the cost per manufactured part of additive manufacturing is typically substantially higher. This, to an extent, is mitigated by the lack of costs associated with tooling (e.g. compared to injection moulding, no mould is needed), but these costs diminish as the number of manufactured parts increase, as would be the case in mass production [15]. This limits the use of additive manufacturing (assuming no other inherent advantages) to limited runs of parts. This increase in cost is primarily due to the cost of the materials used and the energy used in manufacturing the part. The material costs will be largely dependent upon the additive manufacturing technique (e.g. the cost of UV curable resin for stereolithography is substantially greater than a thermoplastic). The energy costs are associated both with the need to have a means of selectively forming a material (e.g. lasers in stereolithography and polymer sintering, a mobile heated extruder in fused deposition modelling) and the increased time needed to make each part in a layer-by-layer fashion.

To address the issue of cost per part it is therefore apposite to look at reduction in material costs, the energy required to process the material and the time taken to produce the part. The easiest way to reduce the time taken to manufacture a part is to coarsen the resolution of the part; this reduces the number of layers that need depositing, but obviously has the side effect of reducing the quality of the part. Another possibility is to reduce the time taken for each layer to be fabricated, which is most readily achievable by using an area-based energy exposure, rather than scanning of a point energy source. Two such methods that have demonstrated area-based exposure are High Speed Sintering, which uses an infra red lamp and a patterned infra red absorber to selectively fuse together polymer powder [16]; and area based stereolithography, which utilises a UV emitting lamp in conjunction with a digital micromirror device as used in most modern projectors. One of the most developed of the area-based stereolithography techniques is Continuous Liquid Interface Production [17]. This technique in addition makes use of an oxygen permeable and UV transparent window through which the UV light is projected. The permeability to oxygen increases the local concentration of oxygen within the resin next to the window, which acts to prevent cross-linking adjacent to the window. This results in a persistent interface between the curable resin and the oxygen depleted zone, at which the curing takes place. As there is resin permanently below the cured layer, issues associated with recoating or 
refilling are eliminated and the curing can therefore be carried out at a far higher rate, with that rate a compromise between time to build part and resolution.

While it is possible to process a wide range of materials via additive manufacturing (e.g. polymers, metals and ceramics), the ability to combine dissimilar materials into the same manufactured component is currently severely limited. This is due fundamentally to the different conditions under which different materials can be processed in bulk to go from feedstock to final part. A relatively straightforward example of this would be the temperatures necessary to sinter metal powder would be significantly higher than the melting and combustion temperatures of a thermoplastic polymer. In addition, there would be issues associated with residual stress, adhesion between materials and thermal expansion mismatch during processing. Another important consideration for the use of dissimilar materials is how to selectively deposit each material. In the case of powder bed processes, the simple action of bulk recoating of the build area with a single powder would have to be adapted so that two or more powders could be selectively deposited on the top of the build area. While some research has indicated that such a system may be technically feasible [18], it would add significant complexity to the process.

One of the primary drivers for using multiple dissimilar materials is to make it possible to embed other functionalities such as electronics within the additively manufactured devices. Due to the complexity of using dissimilar materials with the same manufacturing technique, work has unsurprisingly focussed on the integration of two or more fabrication techniques to produce such a device. To date, the majority of work has involved the integration of an additive manufacturing technique, a means to deposit a conductive interconnect, and a pick-and-place robot for other components. The additive manufacturing techniques used have typically been stereolithography [19, 20] or fused deposition modelling [21, 22], and the conductive traces have typically been fabricated by paste extrusion $[19,23]$ or by ultrasonic wire embedding $[24,25]$. While such processes are capable of producing parts with embedded circuitry of significant complexity, it should be noted that such techniques are typically only viable for prototyping and short production runs. This is for similar reasons as discussed earlier with regards to additive manufacturing techniques, both the conductive traces and the embedded components are produced by a single deposition tool that has to scan the entire deposition path in a vector. For embedded electronics in additive manufacturing to move further towards mass production, it is necessary to identify ways of forming the electronic circuit that is both readily integrated into additive manufacturing, uses processing conditions suitable to typical additive manufacturing materials and is capable of covering large areas quickly so as not to slow down the manufacturing process significantly.

Inkjet printing is an additive and contactless direct write method in which a very small volume $(\sim 1-1000 \mathrm{pL})$ of material-laden ink can be precisely positioned in well-defined patterns [26]. This capability has previously been exploited within additive manufacturing, either by the direct printing of a three dimensional object [27, 28], printing of a binder material into a bed of powder $[29,30]$, or the printing of selective sensitiser onto a powder bed subjected to further processing [16]. In addition to these uses in additive manufacturing, inkjet printing has also been used in areas as diverse as tissue engineering [31], biosensor fabrication [32], aerospace composites [33] and printed electronics [34]. Of particular relevance to additive manufacturing is the field of printed electronics, due to potential for integration of electronic circuits into additively manufactured structures.

An understanding of how a droplet is generated, how a fluid can be optimised for printability, how droplets spread and coalesce on a surface, how evaporation controls the formed solid deposit, and how this solid deposit is processed to become functional (if necessary), is therefore critical in establishing the capability of inkjet printing in conjunction with other additive manufacturing technologies. The rest of this review aims to give a better understanding of the inkjet printing process, covering both fundamental physical aspects of how patterns are formed and the capabilities of the process. Specific attention will be paid to the materials typically used in inkjet printing of electronics, and how the inks used and subsequent processing of the deposits have been developed to be amenable to low temperature polymer substrates of a similar nature to materials commonly used in additive manufacturing.

\section{Droplet generation}

Inkjet printing is an additive and non-contact method of depositing liquids in a computer-controlled pattern. In addition to conventional graphical applications, inkjet printing has been used in many applications that are amenable to solution processing; examples of these include biological scaffolds and cells for tissue engineering [31, 35-37], RFID tags [38], MEMS [39], and photovoltaics [40]. While these applications clearly differ significantly in terms of the final fabricated item, the method by which a droplet is generated, and the constraints placed upon the fluid that can be printed, are similar.

For a droplet to be generated, a pressure pulse must be generated, within an ink-containing vessel, that travels towards an orifice of defined size at one end of the vessel. Upon reaching the orifice, the pressure pulse must be of sufficient magnitude to cause the fluid meniscus at the nozzle to deform and project a ligament of fluid, which is of sufficient kinetic energy to break off from the nozzle and proceed to reduce surface energy by forming a sphere (or spheres). There are two primary ways of generating the requisite pressure pulse: use of a resistive heater placed near the nozzle to generate a rapidly expanding bubble (thermal) [41], or use of a piezoelectric actuator that deforms upon application of a short voltage pulse (piezoelectric) (Figure 1) [42]. While thermal inkjet is used extensively in graphical applications and has been used for functional inks (typically using refurbished graphics printing cartridges) [37, 43], most work that looks to print functional inks and devices has used piezoelectric inkjet printheads [44, 45].

The manner in which droplets are generated in inkjet printing places a number of constraints on the fluid properties that can be deemed printable. The propagation of the pressure pulse through the ink will be attenuated by the fluid, with the degree of dissipation being dependent upon the fluid viscosity [42]. 


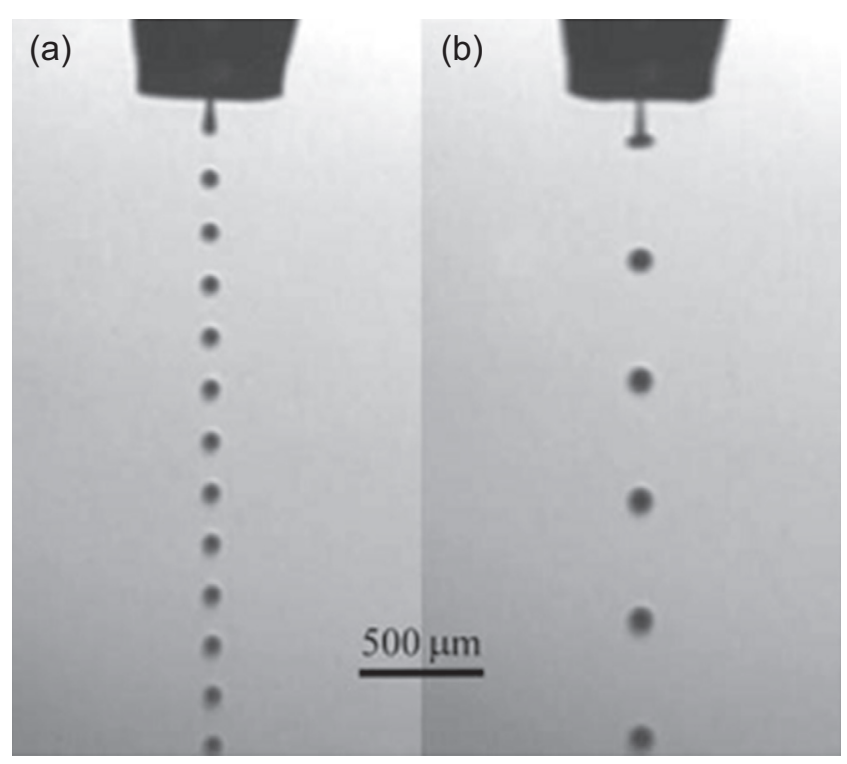

Figure 1. Stroboscopic imaging of silver nanoparticle ink droplet streams obtained at different driving parameters using a piezoelectric drop-on-demand inkjet printer: (a) frequency $=6 \mathrm{kHz}$, pulse duration $=45 \mu \mathrm{s}, \Delta V=50 \mathrm{~V}$; (b) frequency $=6 \mathrm{kHz}$, pulse duration $=13 \mu \mathrm{s}, \Delta V=70 \mathrm{~V}$ [44].

A limit is therefore placed upon the ink viscosity dependent upon the maximum pressure pulse that can be introduced.

Due to the high shear rate encountered in inkjet printing $\left(10^{4}-10^{6} \mathrm{~s}^{-1}\right)$ [46], the non-Newtonian behaviour of the fluid is of importance, with shear-thinning behaviour preferable [47], meaning that any solid material within the ink must be well dispersed and characterised. A further issue observed primarily with polymer solutions is due to a transition under high extensional flow to viscoelastic behaviour. This inhibits printability due to the occurrence of a persistent filament between the droplet and nozzle that inhibits detachment of the droplet from the nozzle [48]. The molecular weight of the polymer is found to be critical in determining the transition between these two behaviours, with higher molecular weight polymers found to bring about viscoelastic behaviour at a lower solid loading. This is due to the increased likelihood of interactions between polymer chains as the chains get longer [49, 50].

The surface tension of an ink is important in the drop generation process as it defines the strength of pressure pulse needed to perturb the meniscus at the nozzle and the ability of the ink to spheroidise after detachment of the ink ligament. These two mechanisms in which surface tension are involved, however, are contradictory, demonstrating the complex relationship that surface tension has on the printability of an ink. In practice, fluids have been successfully printed over a wide range of surface tensions; from organic solvent-based inks with low surface tension $\left(\sim 20 \mathrm{~mJ} \mathrm{~m}^{-2}\right)$ [44] to high surface tension liquid metals $\left(\sim 500 \mathrm{~mJ} \mathrm{~m}^{-2}\right)$ [51].

Numerical modelling [52] has demonstrated the interplay between the fluid properties on the printing process by means of the Ohnesorge number $\left(\mathrm{Oh}=\eta \sqrt{\rho D_{0} \sigma}\right.$, where $\eta$ is the fluid viscosity, $\rho$ is fluid density, $D_{0}$ is the nozzle diameter and $\sigma$ is the fluid surface tension). This number is a ratio of the viscous forces within the nozzle to the surface and inertial forces, and it was derived that this ratio had to be between 0.1 and 1 for successful ejection [52]. From experimental observation this range of fluid properties does successfully print [47], although examples of successful printing have been demonstrated at values below 0.1 when printing with distilled water [53] and polymer solutions [54].

For the printing of solid features, it is necessary for a solid or solid precursor to be present within the ink. It is often desirable for an ink to have as high a solid loading as possible, so as to aid in pattern definition by pinning the contact line or to increase the thickness of deposit. The demand for high solid loading is antagonistic to the viscosity constraint discussed above, as any increase in solid phase (or solute) has the capacity to dramatically increase the viscosity of the ink. When a solute in solution is used, the solid loading is further limited by the solubility of the material in the solvent used. Empirical relationships, such as the Krieger-Dougherty model have shown good agreement with experimental data $[55,56]$ and predict a sharp rise in viscosity above approximately $40 \%$ volume fraction of particles, making any significant increase above this impractical for printing. This volume fraction assumes that the particles in suspension have an aspect ratio approximating unity and are well dispersed. For high aspect ratio materials (such as nanotubes or nanowhiskers), the maximum volume fraction that is printable is significantly reduced [57].

For an ink to print reliably, it is also a prerequisite that their use does not block the nozzle. For dissolved material; this is most likely to occur due to evaporation of solvent at the nozzle (and the solute coming out of solution) [58], and for particles is most likely to occur due to agglomeration of suspended particles at the nozzle or evaporation [59]. Care must therefore be taken in choosing the carrier solvent for inks, with relatively high boiling point solvents often used to counteract this [60].

The resolution achievable by inkjet printing dictates the size of the final printed feature. The higher the resolution attainable, the more complex an electronic structure can be in a given space, and the performance of some components (such as field effect transistors) is directly correlated to the minimum feature size. Combined with the concurrent advantages of reduced materials consumption, there is therefore concerted interest in miniaturising printed devices. For printing, this is generally limited by the minimum size of droplet that can be produced. For conventional droplet generation that relies on the generation of a pressure pulse, this minimum droplet volume that can be generated and accurately deposited is limited to approximately $1 \mathrm{pL}$, which corresponds to a ejected droplet diameter of approximately $12.4 \mu \mathrm{m}$ [61]. While droplet generation below this size is possible using conventional actuation, ballistic accuracy of the droplet is lost, this is due to the kinetic energy of the droplet being insufficient to overcome the air turbulence and drag that acts upon the propelled droplet. To overcome this, it would be necessary to increase the velocity of the droplet, which in turn would require a less viscous ink so that the pressure pulse was not attenuated to the same extent. This requirement for a lower viscosity necessitates lower solid loading of the ink, which is detrimental in terms of the final functioning of the printed device. 
Alternative droplet generation methods have been investigated, which are capable of producing smaller droplets with a significantly higher kinetic energy and therefore maintain ballistic accuracy. These methods impose an electric field between the nozzle and substrate that induce an electrohydrodynamic instability that causes droplet ejection [62,63]. This technology is capable of depositing droplets of sub-femtolitre volume, which corresponds to a sub-micron droplet diameter. This technique has been demonstrated for a variety of materials, such as THz planar metamaterials [64], 3D microbatteries [65] as well as metallic electrode materials [66].

To fabricate printed electronic circuits, particularly on polymer substrates that are not stable at significantly elevated temperatures (such as those likely encountered in additive manufacturing), there are a number of criteria that an ink system must fulfil besides merely having suitable fluid properties. The ink must be compatible with the substrate upon which the circuit is to be deposited, which limits the range of solvents that can be used with some polymers. Any further processing of the ink to obtain a functioning electrode (e.g. heating or chemical treatment) must also be compatible with the substrate. Most importantly, the functioning characteristic of the material (e.g. conductivity or resistivity of a conductor) must be sufficient for the device to function.

\section{Droplet behaviour on a substrate}

Any pattern or structure fabricated by inkjet printing is made up of a series of nominally identical generated droplets distributed according to computer-controlled instruction. It is therefore imperative to understand the behaviour of these droplets, both in terms of interaction with the substrate and each other, to understand the resolution and capability of the inkjet printing process.

The droplet as produced is a liquid, which to form a deposit of use must impinge upon a substrate and change phase. The impingement process should dissipate any excess energy of the droplet upon impact and reach an energetically stable state with both the substrate and any other droplets previous deposited within the locale. The phase change typically takes the form of evaporation of carrier solvent $[38-40,44,54,60$, 67], although can also be via solidification [47, 51, 56], or gelation $[68,69]$. Depending upon the ink system used, it may subsequently be necessary to perform further processing to achieve the desired functionality, such as thermal treatment [44, 70-72], electromagnetic irradiation [73-75], or chemical treatment [76-81].

As mentioned previously, the droplet generated by the printer will have both a kinetic and surface energy dependent upon the fluid properties and the waveform used to produce the droplet. When impinging upon a substrate, the manner in which this energy is dissipated is dependent upon the amount of energy that needs to be dissipated and the nature of the substrate and surrounding environment. These energy dissipation channels can take numerous forms, but can be loosely separated into those that are unstable (e.g. splashing and bouncing) and those that are stable (impact-driven and surface energy driven spreading). While an understanding of unstable impingement is of significant scientific interest (see e.g. a review on the subject by Yarin [82]), it is generally not seen with printing.

Stable spreading has similarly been of significant scientific interest for over a century [83-85] and has typically been investigated using $\mathrm{mm}$-sized droplets, with the findings then made dimensionless using Reynolds number (Re), Weber number $(\mathrm{We})$ and similar dimensionless quantities. These models typically take the form of an energy balance between the initial energy of the droplet and the energy consumed by viscous dissipation to attain the maximum spreading of the droplet upon the surface.

Due to the small size, caution should be taken with trying to relate the dimensionless models derived from $\mathrm{mm}$-sized droplets to the smaller scale printed droplets. With stringent requirements in terms of both temporal and spatial resolution required to directly observe impacting inkjet droplets, comparatively few studies investigating this regime. The first such study by van Dam and Le Clerc [53] demonstrated the need for caution in applying the previously derived models as poor agreement was found between them and observed maximum spreading, with a tendency of pre-existing models to over-predict the spreading. This was subsequently shown to be the case in other studies, and was attributed to the models for mm-size droplets neglecting to include a term that accounted for energy dissipation at the advancing contact line of the droplet $[86,87]$.

Van Dam and Le Clerc [53] also looked at the final diameter of deposits left by ejecting a silver salt solution onto glass, and found the obtained diameter to be largely invariant with impact velocity. This shows that the impact-driven spreading of a droplet does not necessarily dictate the size of deposit formed, with subsequent capillary flow to obtain surface energy equilibrium between droplet and substrate having a significant, and potentially dominant, influence. The influence of surface energy interactions upon the final deposit size of inkjet printed droplets has been modelled by assuming that the droplet will form a spherical cap of equal volume to the initial droplet upon the substrate and that the contact line is pinned, with the contact diameter of the cap $\left(D_{\text {eqm }}\right)$ being determined by the equilibrium contact angle of the ink with the substrate $\left(\theta_{\text {eqm }}\right)$. This can be normalised to the initial droplet diameter $\left(D_{0}\right)$ to obtain a spreading ratio, $\beta_{\text {eqm }}$ [53]:

$$
\beta_{\text {eqm }}=\frac{D_{0}}{D_{\text {eqm }}}=\sqrt[3]{\frac{8}{\tan \frac{\theta_{\text {eqm }}}{2}\left(3+\tan ^{2} \frac{\theta_{\text {eqm }}}{2}\right)}}
$$

This relationship (Eq. (1)), and similar, has been shown to reliably predict the diameter of individual printed deposits $[88,89]$.

For the liquid droplet to reach equilibrium, it necessitates that the droplet does not undergo significant phase change during the spreading process. Any phase change (such as solidification, gelation or evaporation) may lead to either a change in droplet/substrate equilibrium or a premature arrest of the contact line before equilibrium is reached. Such occurrences have been seen in solidifying systems [90], evaporating systems [91], and gelling systems [69], typically by variation of the 
substrate temperature. While use of temperature variation can be used to control the dimensions of a printed deposit, it is not common to do so with inks having a significant solid loading due to detrimental effects on the reliability of printing, primarily due to an increased likelihood of nozzle clogging.

For inks that rely on evaporation to change phase from liquid droplet to solid deposit, the deposit size is largely dictated by the surface energy interactions between the ink and the substrate and the initial volume of the ejected droplet. This is despite the volume of the droplet decreasing over the evaporation process, which one would reasonably expect to lead to a reduction in the droplet footprint to maintain surface energy equilibrium. While such a reduction in size has been seen for some printed systems [92], it is generally not the case. This is due to an energetic barrier being present that resists retraction of the contact line, often referred to as contact angle hysteresis [88]. This hysteresis may be caused by surface roughness [93], chemical inhomogeneity [94], or the presence of a second solid phase such as dust contamination [95]. In the case of solid-laden liquids, the evaporation process itself will lead to the manifestation of a solid phase that can act as a barrier to retraction of the contact line, and is often sufficient to prevent any recession of the contact line over the entire evaporation process (referred to as a zero receding contact angle) [88].

The prevalence of ink and substrate combinations that manifest a zero receding contact angle in part leads to another often observed phenomena; that of coffee staining (Figure 2); coffee staining constitutes the preferential deposition of solid material at the periphery of a droplet (or any other arbitrary footprint of liquid on a surface) [95]. The necessary preconditions for coffee staining are that the droplet has a non-zero equilibrium contact angle with the surface, the contact line is pinned and that evaporation occurs [96]. The evaporation of the droplet drives the segregation of material due to the differences in surface area to volume ratio between the edge of the droplet and at the centre, with the ratio being higher at the edge and lower in the centre. The loss of solvent from the droplet due to evaporation will be relatively invariant over the free surface of the droplet (although the rate may be greater at the droplet edge [96]), but a pinned contact line will mean that the droplet is unable to uniformly change volume. It is therefore necessary for solvent to be transported from the centre of the droplet to the periphery, with this flow being sufficient to overcome any forces between the dissolved or suspended phase in the droplet and the substrate, and therefore depositing material at the contact line $[96,97]$.

The generation of flows within the droplet due to the necessity of mass transport can also lead to the formation of other morphologies depending upon the nature of the flow, such as segregation of material in the centre of the droplet [98]. It is, however, generally desirable for there to be an even distribution of material over the wetted area, particularly for conductive material and layered structures. To achieve a relatively even distribution, and therefore counteract the propensity for coffee staining, it is necessary to disrupt the outward flow that causes the segregation of material. This has been demonstrated in a number of ways such as generating a contrary Marangoni flow due to surface tension and temperature gradients across the droplet surface [99], varying the temperature of the substrate to reduce the evaporation rate at the droplet periphery [100], introducing an additional flow due to capillary action into (a)

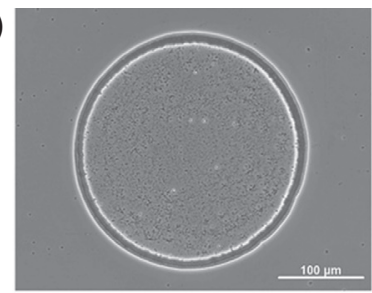

(b)

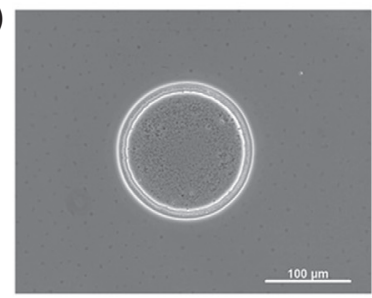

(c)

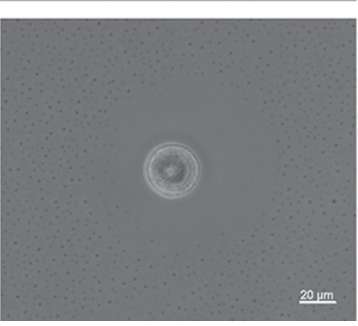

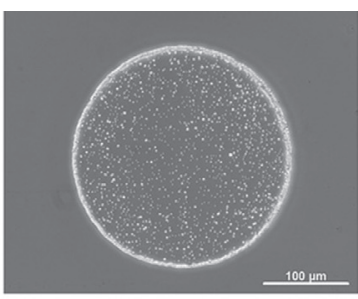
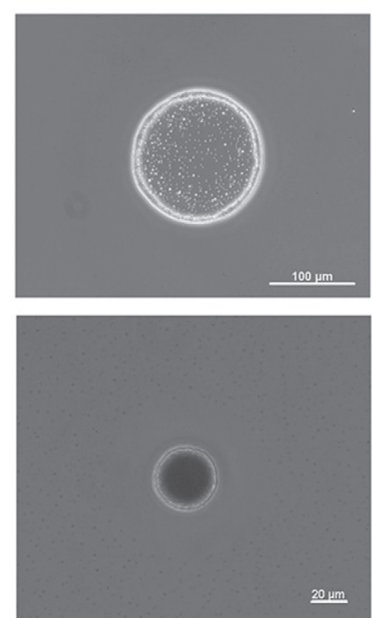
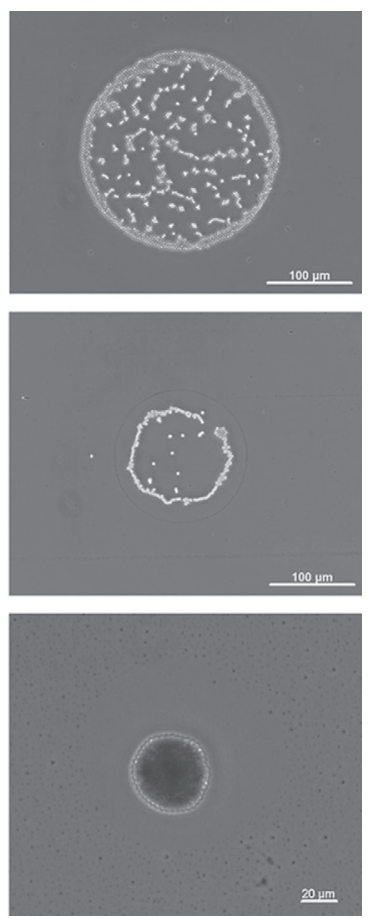

Figure 2. Phase contrast microscopy images of inkjet printed droplets of aqueous suspensions of silica particles on high surface energy substrates (a), cleaned glass slides (b), and low energy substrates (c). Each row shows, from left to right, a particle size of $0.33 \mathrm{~mm}, 1 \mathrm{~mm}$ and $3 \mathrm{~mm}$, respectively [97]. 
the substrate [101], preventing the contact line from pinning [102], increasing the attractive force between suspended particles and the substrate [103], and by introducing an additional solvent of low vapour pressure [104, 105].

To form 2-dimensional patterns from printed droplets. It is necessary to either print droplets at such a rate that they undergo phase change before any subsequent adjacent droplets are deposited, or for the droplets to coalesce in the liquid state. Due to the timescales involved in the phase change, especially for evaporation, it is generally preferable for the droplets to coalesce in the liquid state for the sake of printing speed. If the deposition frequency is such that the a previously deposited droplet has undergone phase change before any subsequent droplet is deposited, a 'stacked coin' morphology is formed [100]. This is, in essence, a series of closely overlapping circular deposits from individual droplets. In addition to the issues with printing speed mentioned previously, producing linear features in this way will tend to produce a non-flat topography due to any subsequent droplets being deposited upon a non-flat surface (i.e. the previously deposited droplet). Due to the circular nature of an individually deposited droplet, the contact line is made up of a series of arcs rather than being truly straight, with this exacerbated as the droplet spacing is increased.

To produce a feature with a constant cross section (or "uniform" morphology), it is necessary for the droplets to coalesce in the liquid state. Furthermore, it is necessary that there is sufficient contact angle hysteresis that there is no recession of the contact line upon coalescence to minimise surface energy [88]. As mentioned previously, this behaviour is quite prevalent in the ink and substrate combinations used to fabricate conductive patterns. These two prerequisites for the production of constant cross-section features enable a straightforward volume balance between the deposited droplets and a stable bead with a cross section of a circular segment, as in Figure 3 [44]:

$$
\frac{\pi D_{0}^{3}}{3 p}=\frac{w^{2}}{2}\left(\frac{\theta}{\sin ^{2} \theta}-\frac{\cos \theta}{\sin \theta}\right)
$$

Where $w$ is the width of the bead and $p$ is the spacing between each deposited droplet. From this volume balance (Eq. (2)) it is possible to obtain a prediction of the bead width as a function of printing parameters and contact angle and has been verified experimentally [44, 88, 89, 100, 105].

Due to the zero-receding contact line condition necessary for this volume balance, it is inherent that the minimum feature size attainable will be that of a single deposited droplet. This therefore necessitates that the minimum width of bead for which equation (2) is valid is equal to $\beta_{\text {eqm }} D_{0}$ as defined in equation (1). Using this condition, it is possible to define a critical droplet spacing, $p_{\max }$, above which there is insufficient deposited liquid to form a stable bead with parallel contact lines:

$$
p_{\text {max }}=\frac{2 \pi D_{0}}{3 \beta_{\text {eqm }}^{2}\left(\frac{\theta}{\sin ^{2} \theta}-\frac{\cos \theta}{\sin \theta}\right)}
$$

A droplet greater than the value of $p_{\max }$, but smaller than $\beta_{\text {eqm }} D_{0}$ will result in a periodic curvature to the contact line

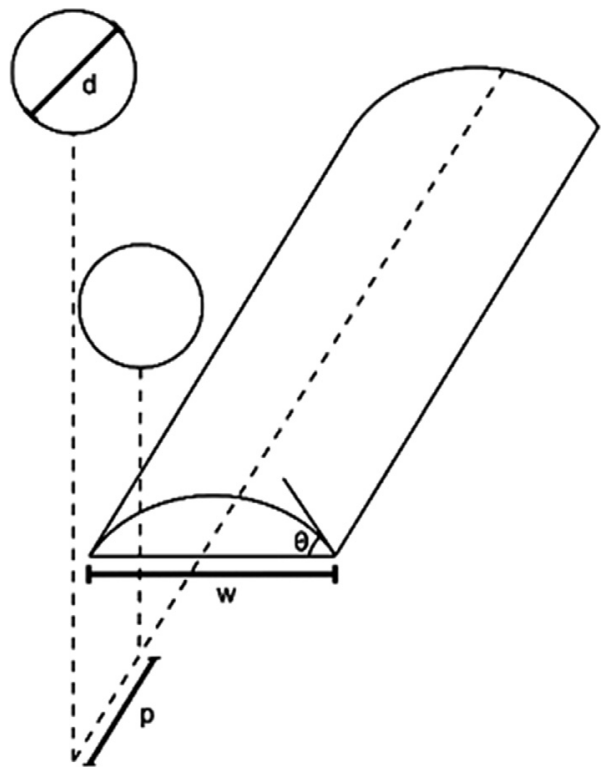

Figure 3. Diagram showing the parameters that are used in modelling the relationship between final track width and contact angle [44].

due to the insufficient volume of liquid, which is referred to as a "scalloped" morphology [100].

A final morphology observed in printed lines is that of "bulging" [68, 88, 89, 104, 106]. This was first observed by Duineveld [88], and was explained due to the relative driving forces for flow of newly deposited droplets. A newly deposited droplet will have a smaller radius of surface curvature than a pre-existing bead of liquid with which it coalesces. This difference in curvature results in a pressure difference, with newly deposited liquid moving from the front to the interior of the bead, resulting in the bead front having a contact angle below the advancing contact angle and preventing any spreading. For this axial flow to be significant in determining the final morphology, it is necessary for it to be of sufficient magnitude compared to the applied flow to the ridge due to newly deposited droplets, as there will otherwise be insufficient time for the axial flow to take place for the contact angle to be suitably reduced.

Duineveld [88] used such arguments to construct a mathematical model of the bulging process that agreed well with experimental results, showing that occurrence of bulging was exacerbated by decreasing deposition frequency (reducing the applied flow rate), increased ink/substrate contact angle, and reducing droplet spacing (both increasing the driving force for axial flow). By adapting this model and allying it to the volume conservation balance given in equation (2), it was possible to produce a stability map [106] that enabled the prediction of line morphology based upon ink properties, printing conditions and ink/substrate interactions (Figure 4). This map has subsequently shown good agreement with experimental results for a wide variety of ink/substrate combinations [104, 106, 107].

The aforementioned factors that control line morphologies and deposit segregation of single droplets can be extended to films, with 2 solvent mixtures found to be critical in obtaining 


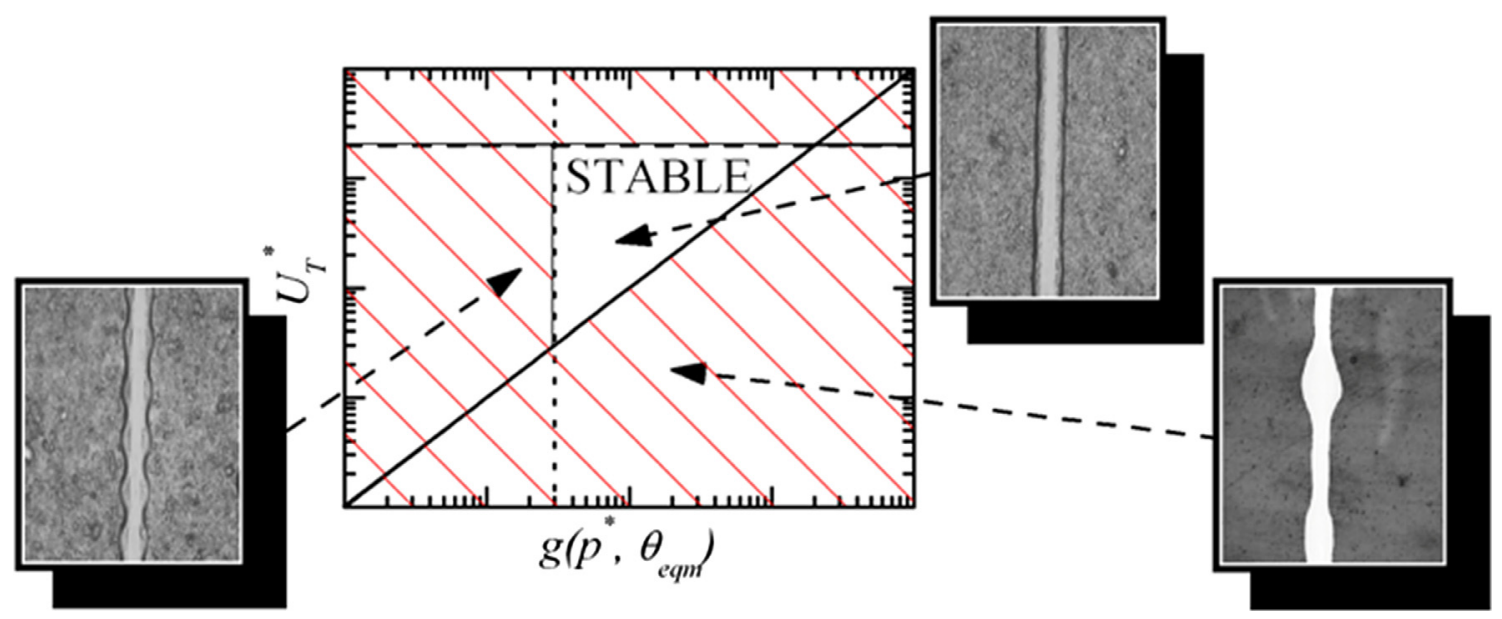

Figure 4. A stability map for inkjet printed tracks, showing the triangular region of stability in the centre, the "scalloped" region to the left and the "bulging" region in the bottom right [106].

topographically smooth films [108]. It was also found that the homogeneity of these films was improved by printing droplets in a quasi-random order and at low speed [108], with the improvement in homogeneity of the films most likely due to the elimination of pressure gradients within the film caused by newly deposited liquid and evaporation. This was expanded upon by Soltman et al. [109], where the variation in the contact angle of the printed film due to flow and evaporation were numerically modelled. This led to the use of variable line spacing so that the contact angle was kept between the advancing and receding contact angle. The need to keep the contact angle of the liquid film with the substrate between the advancing and receding contact angle has also led to the use of 2 solvent mixtures [110] and controlled surface roughness [111].

The texture of the surface onto which the ink is deposited is of particular relevance to additive manufacturing. For powder bed processes, the fused layer onto which ink could potentially be deposited is likely to have a roughness of similar magnitude to the powder, and in addition is likely to have some residual open porosity. This combination of roughness and porosity will most likely lead to ink being drawn into the powder bed by capillary action. While in some applications this can be viewed as advantageous (for example, printing of a binder material into the powder bed), for printed electronics it may be detrimental due to the possibility of the deposit being discontinuous. For additive manufacturing processes that form a definitively solid layer, such as fused deposition modelling and stereolithography, this is less of an issue. Of more concern, however, is the typically low surface energy of the substrate that could lead to the ink not wetting the substrate satisfactorily. This again raises potential issues with regards to the continuity of any deposits, which is of critical importance in printed electronics. For example, any discontinuity in a printed conductive trace will mean that the whole circuit would cease to function. Strategies to overcome similar problems in conventional printed electronics have been demonstrated, with the substrate being treated to selectively vary the wettability (e.g. by plasma treatment [112] or laser treatment [113, 114]), structuring the substrate to introduce a geometric confinement to the ink [115], or a combination of the two [67]. It is feasible that both of these techniques or similar could be integrated into an additive manufacturing process, with either the use of a further deposition head (in the case of surface treatment) or creation of physical barriers using the additive manufacturing technique.

As an essential component of any electronic circuit, a conductive trace is unsurprisingly one of the most studied components in printed electronics. A number of different materials for use as a conductor have been studied such as conducting polymers $[115,116]$, and carbon allotropes [117, 118]; however, the most studied group of conductive materials in printed electronics have been metallic. This is due to their combination of low resistance, environmental and mechanical stability and relatively low cost. The next section will focus on the use of metallic inks in printed electronics, with particular focus on strategies that have been developed for their processing that enable the use of temperature sensitive polymer substrates similar to the materials used in additive manufacturing.

\section{Metallic inks}

Most contacts and interconnects that have been inkjet printed have used inks that have a metal (e.g. silver) as their chief functional component, which is due to the significantly higher conductivities that can be obtained for the final printed feature. There are two types of metal-containing ink that can be used. The first type is composed of a suspension of stabilised nanoparticles and a carrier solvent, and is known as a nanoparticle (NP) ink. The second type has a metal salt dissolved into a suitable carrier, and has often been described as a metalorganic decomposition (MOD) ink [43, 69, 119].

Both ink types have their respective advantages and disadvantages. MOD inks, a simpler type of ink due to being solutions, do not require colloidal stabilisers and nozzle clogging is reduced. Although MOD inks tend to have lower loadings, which means less metal is deposited with each pass, the 
conductivity that can be obtained is high; values exceeding $50 \%$ bulk silver have been obtained for single-layered tracks using a cure time of $5 \mathrm{~min}$ and temperature of $150^{\circ} \mathrm{C}$ [120].

NP inks, on the other hand, have a higher particle loading and are generally more available. NP inks have also been reported as having lower contact resistances [71]. Both inks rely on the reduced melting point of nanoparticles to form conductive features; silver nanoparticles melt at a much lower temperature than bulk silver due to the high surface area to volume ratio [121]. In NP inks, the nanoparticles are already present. Heat is typically required to burn off the surfactant, the nanoparticles then sinter together. With MOD ink, the nanoparticles are formed in-situ.

With both MOD and NP inks, the choice of metal is determined by a number of factors. Cost, obviously, is an important factor. However, the ease of processing and final conductivity tend to be the main considerations, as they determine the end device's functionality. Ideally, a metal-containing ink would have a long shelf life, be straight-forward in terms of jetting behaviour, deliver high levels of conductivity and be affordable.

In terms of conductivity, silver is favoured due to it having the highest, $6.30 \times 10^{7} \mathrm{~S} / \mathrm{m}\left(\rho=1.59 \times 10^{-8} \Omega \mathrm{m}\right)$. Copper $\left(1.68 \times 10^{-8} \Omega \mathrm{m}\right)$, gold $\left(2.44 \times 10^{-8} \Omega \mathrm{m}\right)$ and aluminium $\left(2.82 \times 10^{-8} \Omega \mathrm{m}\right)$ have similar values. The high price of gold immediately discounts it from any bulk applications, and copper requires a special processing atmosphere; Moon et al. reported on the inkjet printing of a copper MOD ink, which was converted in a $3 \% \mathrm{H}_{2}$ atmosphere [72]. To date, silver has been the metal that has been most reported, as it ease of processing offsets its price compared to gold and copper.

However, the cost of copper is encouraging researchers to explore new ways of manufacturing the inks that increase its ease of processing. Magdassi et al. synthesised nanoparticles that were composed of copper core and covered with silver shells; this approach allowed the nanoparticles to be printed and processed in air [45].

\section{Processing of metallic inks}

Whether an NP ink is printed or an MOD ink, both types require a post-printing process step to convert the ink into a conductive, metallic track. Typically, thermal treatments are used. In NP inks, the thermal step decomposes the colloidal stabiliser, drives off the carrier solvent via evaporation, and provides energy to enable the nanoparticles to sinter together. With MOD inks, heat drives evaporation of the carrier solvent, causing the metal salt to precipitate out. Continued heat causes the organic component of the salt to decompose, leaving precipitated and sintered nanoparticles.

There are a variety of methods that are used to encourage sintering. The most widely reported has been conventional heating, in which the substrate that contains the printed feature is placed above a heat source, such as a hot-plate. However, the typical temperatures $\left(\sim 20{ }^{\circ} \mathrm{C}\right)$ that are used in this step tend to favour the use of expensive substrates such as polyimide, and as such are not suitable for use in additive manufacturing. Alternatives to conventional heating include using a laser to sinter [73, 122], however, conductivity is adversely affected by write speed. Another method of introducing the energy needed to enable sintering is the application of an electric field [123], which relies on the pre-sintered nanoparticles forming a slightly conductive network. Upon application of an electric field, resistive heating of this network takes place, which drives off any stabilisers and sinters the particles together. This process is very quick ( $\sim 100 \mathrm{~ms})$, leads to minimal heating of the substrate and leads to conductivity approaching bulk metal [124], but requires a direct connection to a power source, which would potentially be a limitation in additive manufacturing.

Use of microwave radiation has been investigated, and has been found to be capable of producing conductivities of $\sim 5-10 \%$ of bulk metal in under 5 min without damaging polymeric substrates [125]. Due to the penetration depth of microwaves into metals, the use of microwave radiation is limited to features of a few microns thickness [125], although this thickness is sufficient for most sensing applications. The applicability of microwave curing to a pre-existing additive manufacturing process, however, would present a significant challenge.

The use of chemical agents to remove any stabilising agent from the metal has also been investigated, both for MOD inks [76-78] and for NP inks [79-81]. The use of chemical agents reduces or potentially eliminates the need for any thermal treatment that may be detrimental to polymer substrates (Figure 5). In the case of MOD inks, hydroquinone was used as a reducing agent after brief exposure to UV radiation to reduce the silver ion of deposited silver neodecanoate to atomic silver, with conductivities found to be in the region of $\sim 10 \%$ that of bulk silver [76]. Other examples of chemical reduction include the use of silver nitrate with ascorbic acid [77], and a silver ammonia solution reduced by formaldehyde [78]. Chemical sintering has also been used with NP inks, where silver nanoparticles treated with solutions containing chloride ions have been found to result in conductivities approaching that of bulk silver [79-81]. This is attributed to the chloride ions destabilising the stabiliser on the nanoparticle surface $[79,80]$ and simultaneous dissolution/deposition of silver ions at the nanoparticle surface leading to greater interconnectivity [81].

Of growing importance has been the adoption of flash light sintering, also known as photonic sintering [74, 75]. In this technique, a series of short ( $\sim \mathrm{ms})$ flashes from a Xenon lamp irradiates the printed feature. The process is fast, which is attractive for manufacturers, and conductivities of approximately $10-20 \%$ of bulk metal obtainable. Due to the short timescales, this has proved successful with more reactive metals that are not easily processed without controlled atmosphere environments, such as copper [74] and nickel [75]. It should be noted, however, that both the initial capital cost of such photonic sintering equipment and the running costs are significant. This would mean that such equipment is only suitable for applications where the speed and material compatibility advantages outweigh the cost. As such, the cost benefits of using less expensive raw materials are negated until large-scale production is adopted.

For some applications, the deposition of interconnects may be sufficient for embedded electronics in an additively 

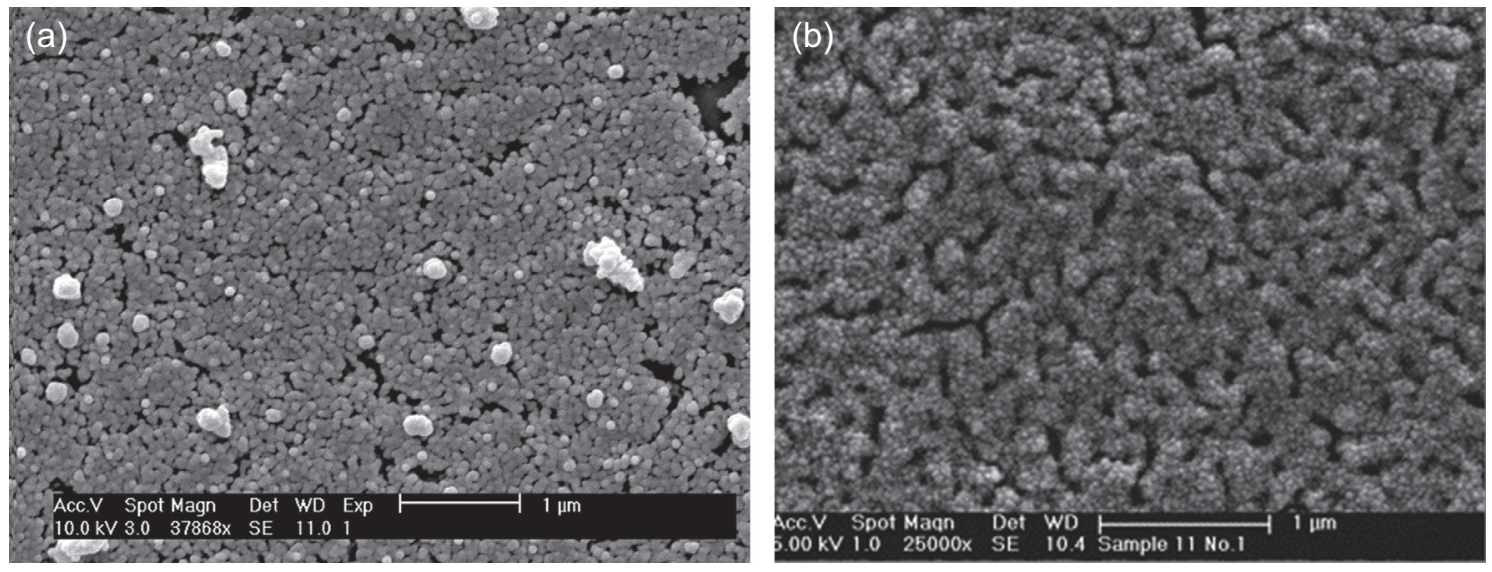

Figure 5. Two SEM images, showing the dense nanoparticle structure obtained using an MOD ink followed by (a) chemical conversion or (b) thermal conversion to obtain silver [76].

manufactured part if combined with the inclusion of pre-fabricated electronic components. There is potential, however, for a more complete printing of the electronic circuit, with the inclusion of printed logic circuits directly into the part. Due to the necessity to produce these components in solution and at temperatures not greatly elevated above ambient, work to date has tended to focus on organic semiconductors, which will now be briefly discussed.

\section{Organic semiconductor inks}

While the embedding of conductors and similar passive components into additively manufactured components is of import by itself (for example, in connecting other electronic components or electromagnetic shielding), the ability to embed active components based upon semiconducting material, such as transistors, introduces far greater design possibilities. The materials typically used for such devices (e.g. silicon crystals) do not lend themselves readily to solution processing, although this has been demonstrated with the decomposition of suitable precursors [126]. It should be noted, however, that the temperatures necessary to form polycrystalline silicon using this route is significantly higher than the melting, glass transition or combustion temperatures of materials typically used in additive manufacturing.

For printed electronics within additively manufactured components, the requirement is that the semiconducting material must also be solution-processable at temperatures amenable to the other (typically polymeric) materials used in the additive manufacturing process. Such requirements are similar to those for printed electronics on flexible substrates, with the bulk of work to date in the field focusing on organic semiconductor materials. To aid in understanding the challenges involved in printing organic semiconducting devices, there now follows a brief discussion of a typical organic semiconductor device and the physics that govern the behaviour of the device.

A typical organic semiconductor device is an organic field effect transistor (OFET), a typical structure is shown in Figure 6. The structure of OFETs consists mainly of source

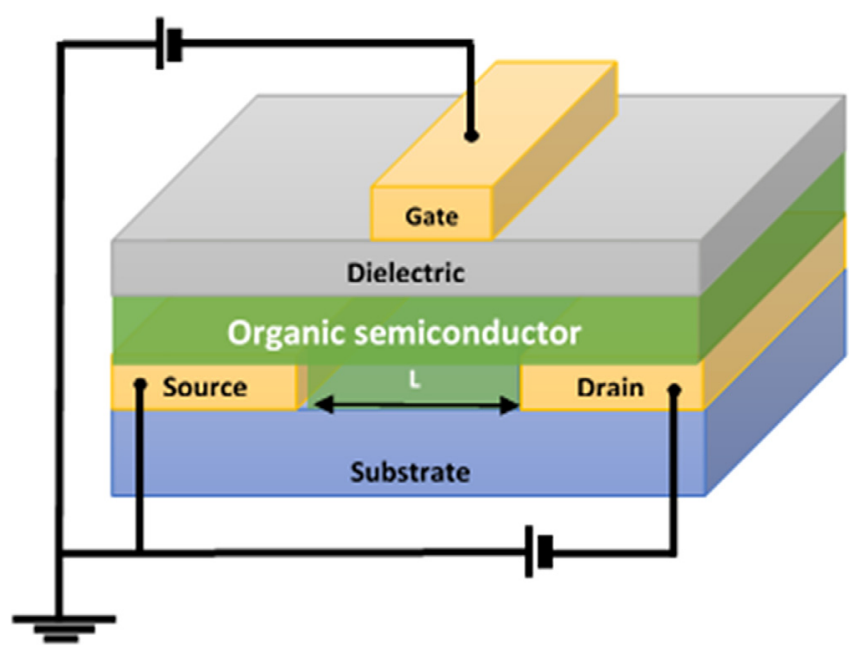

Figure 6. A typical OFET structure, showing the location of the source, drain and gate electrodes.

and drain electrodes separated by a distance (channel length, $L$ ). An organic semiconductor (OSC) layer bridges the source and drain electrodes. Over the OSC layer is a dielectric (either dry, analyte or gel insulator) upon which there is a gate electrode. The actual structure of an organic thin film transistor varies and several architectures have been reported [127].

An OFET is a voltage-operated device since the current can be introduced into the organic semiconductor channel by a voltage applied to the gate electrode. Consequently, the current flow through the channel can be changed and controlled by the gate voltage. This feature allows OFETs to operate as a type of amplifier or a transducer through converting a voltage or potential signal into a current response [128]. More details of the principles, understanding the structural, and electrical properties of materials used to construct OFETs have been reviewed elsewhere [128, 129].

Line width, channel length, and uniformity of OFET electrodes are some of the most essential factors to consider in order to produce high current. The metal used in the source 
and drain electrodes should provide a good injection of charge carriers to the organic semiconductor. High work function electrodes are required for injecting the charge carriers to a hole (positive) organic semiconductor. The distance between the source and drain electrodes (channel length $L$ ) should be very short, according to the following equations, since the drain current is inversely proportionate to channel length, $L$.

Within the linear regime, the current can be expressed as:

$$
I_{D S}=\frac{W}{L} C_{i} \mu\left(V_{G}-V_{T}\right) V_{D} \quad \text { for } \quad\left|V_{D}\right|\left|V_{G}-V_{T}\right|
$$

Within the saturation region, the current is defined:

$$
\begin{gathered}
I_{D S}=I_{\text {sat }}=\frac{W}{2 L} C_{i} \mu\left(V_{G}-V_{T}\right)^{2} \text { for } \\
\left|V_{D}\right| \geq\left|V_{G}-V_{T}\right|
\end{gathered}
$$

Where $\mu$ is the mobility, $C_{i}$ is the capacitance per unit area of electrolyte, $V_{G}$ is gate voltage, $V_{D}$ is drain voltage, $V_{T}$ is threshold voltage, and $W$ and $L$ are the width and length of the channel, respectively.

In the linear regime, the drain current, $I_{D S}$, rises linearly with drain voltage, $V_{D}$, while the saturation region shows the drain current, $I_{D S}$, remains constant at a saturation level $\left(I_{\mathrm{sat}}\right)$ even if the drain voltage, $V_{D}$, is increased further. The channel current strongly depends on the channel length. Since, the current increases with shorter channel length. Furthermore, the switching on/off time-speed is inversely proportionate to the square channel length $(L)$.

To achieve the best performance of an organic semiconductor (i.e. highest $I_{D S}$ ) in a device such as an organic field effect transistor (OFET), the following are therefore required: a high degree of surface morphology of the organic semiconductor (crystallinity, with greater crystallinity increasing mobility, $\mu$ ), good quality of contacts for injection of charge, and a high dielectric capacitance $\left(C_{i}\right)$. The importance of the materials used for the organic semiconductor thin layer in an OFET represents one of the essential elements in the production of devices with highest performance and environmental stability. Hence, much effort in the synthetic chemistry and physical chemistry fields is aimed at producing high mobility materials. Importantly, the performance of organic devices is improved as the crystallinity of the deposited organic semiconductor thin layer increases. Single-crystalline organic thin-film transistors have displayed distinguished characteristics such as higher mobility and on/off current ratio [130, 131]. The solvents used for dissolving organic semiconductors play a critical role in controlling the maximum solution concentration and rate of solution evaporation, which in turn leads to the production of a crystalline thin film. For example, the use of a high boiling point solvent is recommended because its evaporation rate allows the semiconductor more time to crystallise, and as a result, a high mobility can be obtained.

A variety of methods have been used to produce organic single crystals such as vapour deposition methods and solution based processes. However, some of these methods, in particular spin coating, have shown negative aspects such as significant material consumption and substrate contamination, which limits the control of spatial selectivity and film uniformity [132]. In order to overcome such limitations, inkjet printing has been considered as a promising technique for preparing organic single-crystal transistor arrays as a result of its ability to dispense only the required amount of material (thereby reducing both waste and cost) and to its highly selective patterning nature [133]. However, using inkjet printing without due consideration to produce highly crystalline thin films may result in issues such as self-organizing of the deposited materials which produces films with a non-uniform thickness distribution, difficulties in ink formulations, and complex drying phenomena such as coffee staining $[132,133]$. Single-crystal thin-films for both polymer and low molecular weight organic semiconductors have been prepared by utilizing inkjet printing $[134,135]$. One of these methods is characterized by combining the process of anti-solvent crystallization and inkjet printing. Minemawari et al. found that scalable solidification of organic semiconductor film can be controlled through using the anti-solvent crystallization method and double shot inkjet printing (DS-IJP) technique [134]. This method involves using two types of ink; anti-solvent ink (a liquid in which a substance is insoluble) and solution of a semiconductor in a solvent that is miscible with the anti-solvent. By using piezoelectric inkjet, the anti-solvent ink is printed first and then overprinted with the solution semiconductor ink, which results in a mixture of the semiconductor solution with the anti-solvent. In the first stages of the formation of the film, small floating particles form on the liquid's surface and act as nuclei for ongoing crystallisation. The nuclei experience successive growth, which leads to larger particles being formed. Lines can be perceived on the surface of the tiny drops when they begin to evaporate, which shows the solid nature of the finite product. Minemawari and his group also believe that reversing the deposition sequence between semiconductor solution and the anti-solvent play an important role for obtaining uniform films with high crystallinity when they used different organic semiconductors such as TIPS-pentacene, instead of C8-BTBT semiconductor, as shown in Figure 7 [135].

OFETs with the printed single crystalline thin films as an active layer showed extremely high performance and mobility, $16.4 \mathrm{~cm}^{2} \mathrm{~V}^{-1} \mathrm{~s}^{-1}$ on average. The crystal nucleation and the following growth of crystal domains could be controlled using a suitable design of the droplet configuration. Since they found that the film surface morphology of a shape with a protruded area appears to be more uniform and smoother than the films obtained with a rectangular shape. A stable design of the droplet configuration can be obtained by the predefined wetting patterning. Additionally, printing conditions such as substrate temperature, the concentration and volume of the solution, and the solution-anti solvent ratio play an important role for controlling the morphology of the films as well as their single-domain nature.

\section{Manufacture of complete devices}

As discussed previously, the use of inkjet printing to embed electronic functionality into additively manufactured parts is compelling due to the combination of limited material waste, 

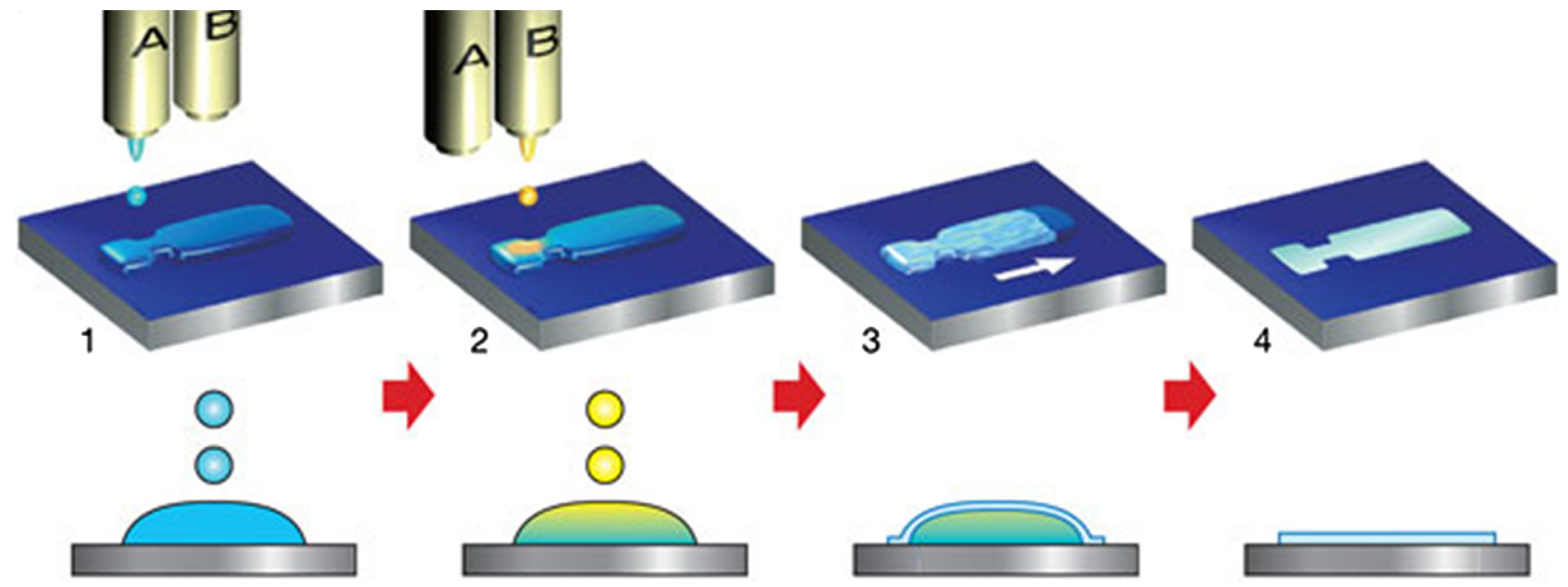

Figure 7. Inkjet printing process of organic single-crystal thin films. The top half of the image shows the reverse sequence between semiconductor solution and the anti-solvent; DS-1 (left) and DS-2 (right). In the bottom half of the image, the anti-solvent ink (A) is first inkjet-printed. The solution ink (B), which contains the semiconductor is overprinted sequentially to form intermixed droplets (step 2). Semiconducting thin films grow at the liquid-air interfaces of the droplet (step 3) before the solvent fully evaporates (step 4) [134, 135].

non-contact deposition to reduce contamination and scalability to large volume production. The last of these points is perhaps best illustrated by graphical printing, where the same fundamental technology is used in a standard A4 desktop printer and much larger roll-to-roll industrial printers capable of printing multiple $\mathrm{m}^{2} /$ minute. The printing of functional components, however, is inherently more complex than graphical printing due to the multi-layer structure of the final device, the post-processing required and the far more critical nature of repeatability and reliability.

The need to build up a multi-layer structure introduces complications, particularly associated with deposition. The dependence of deposit morphology upon the surface energy and topography of the substrate has already been discussed, and it is high likely that a pre-deposited layer will have different surface properties to the underlying substrate. This would be of particular concern if the printed film was not being deposited onto a single material, but on to a multitude such as if the source and drain electrodes of the OFET are being covered with a dielectric or semiconductor layer. SanchezRomaguera et al. demonstrated that this change in morphology can be pronounced, with the change in surface energy between a glass substrate and previously deposited silver track being sufficient to dramatically alter both the width of the line and the topography of a deposited dielectric film [136]. To help counteract the difference in surface energy, it is possible to alter the energy of the surface so that a better deposit is obtained, which is typically done using a UV-Ozone plasma treatment [136].

For multi-layer fabrication, it is necessary to exercise prudence when selecting inks, with particular attention paid to the solubility of previously deposited layers in the solvent of the next ink to be deposited, so as to avoid redissolution. Any damage to previously deposited layers may will be uncontrolled, leading to an uneven layer, which could result in inconsistent device performance or even pinholes in the film and consequent failure of the device. The requirement of a defect-free layer of uniform thickness has led to the use of curable resins as inks, rather than dissolved polymers [136, 137]. These resins, typically cured by UV light, are preferred due to the entirety of the ink being converted to solid, reducing any solute segregation (coffee staining), producing pinhole free layers, and being chemically inert after curing and hence resistant to redissolution.

For large scale manufacture, it is important to bear in mind not only the printing speed but also the time required for any post-deposition processing of each layer. As shown when discussing metallic inks, this processing can take a significantly longer time than the printing itself and each layer may have to be processed after deposition. To expedite this, it is necessary to select a post-processing technique for each layer that is capable of endowing the desired function on the layer (e.g. a minimal sheet resistance), does not cause damage to previously deposited layers and can be performed in as short a time as possible. The use of bulk thermal heating is problematic due to the time required and the potential for damage to polymeric layers, either by melting or by the introduction of thermal stresses that can lead to debonding and cracking of the layers [136]. If electromagnetic radiation, such as flash sintering or UV curing, is used, the degradation of previously deposited layers when subjected to such radiation needs to be identified.

Compared to graphical applications, the repeatability and reliability of printing is paramount in a functional manufacturing process. For a graphical application, a single misdirected or non-ejected droplet out of several million will be barely noticeable. For printed electronics applications, such an occurrence could lead to either a pinhole in a film or a gap within a conductive feature, leading to failure of the device. To ameliorate against such occurrences, it is common to have significant redundancy within the printing process. This redundancy takes the form of high-speed machine vision apparatus that is able to identify any locations where a droplet has not been successfully deposited, followed by a second identical set of print heads that fill in the identified areas. 


\section{Conclusions}

Additive manufacturing, an umbrella term for a number of different manufacturing techniques, has attracted increasing interest recently for a number of reasons, such as the facile customisation of parts, reduced time to manufacture from initial design, and possibilities in distributed manufacturing and structural electronics. Current limitations of additive manufacturing are associated with the time and cost needed to produce a part, the resolution attainable by the additive manufacturing technique and the limited capability to fabricate parts from dissimilar materials. Addressing the time and cost to produce a part has seen additive manufacturing move towards area-based techniques that are to form an entire layer of a part at once. The use of dissimilar materials has also been keenly researched, with particular emphasis on embedding of electronic component and circuitry. The techniques investigated for this have typically relied on a combination of a single nozzle deposition of conductive material in combination with a pick and place robot to position more complex components. To both minimise the time and cost of a manufactured part, as well as enable the use of dissimilar materials, it would be ideal if a deposition technique that can both be easily integrated with other additive manufacturing technologies and was able to deposit dissimilar materials of interest in an area based manner would be ideal. One technique that has the capability to meet these requirements is inkjet printing, which has seen a great deal of research, particularly with regards to printed electronics.

Inkjet printing is a process that dispenses droplets that are uniform in terms of size, and selectively deposits these droplets in an arbitrary pattern on a surface. In doing so with one or more ink loaded with functional material (or a precursor), it is possible to form functional components such as electronic circuits. Formulation of a functional ink is a balancing act between having suitable fluid properties to allow droplet formation, and sufficient loading of material to produce the desired component. Printability of an ink is typically constrained by the viscosity of the ink (both Newtonian and non-Newtonian), which rises as the amount of material dissolved or dispersed within it increases. Numerous strategies have been deployed to overcome this dichotomy, such as the use of nanoparticles, more printable precursors and in situ reactions of multiple materials.

To achieve the desired pattern, it is necessary to understand the how the droplets will behave on the given substrate before forming the final deposit. To minimise surface energy, a liquid on a surface will tend towards a spherical cap. While for some applications a spherical deposit of ink may be sufficient, to achieve other shapes such as lines or rectangular films it is necessary for the movement of the contact line of the ink with the substrate to be constrained. This can either be by engineering the substrate to confine the ink, or by having sufficient solid constituent within the ink to pin the contact line upon drying. The pinning of the contact line can lead to segregation of solid constituent with the ink footprint, which is referred to as coffee staining. This segregation can either be exploited to obtain finer features, or can be minimised by changing the formulation of the ink, the substrate, or the conditions under which evaporation takes place.
Once printed in the desired pattern, it is often necessary to subsequently process the deposited material to form the final functional structure. Of particular focus in this review is the combinations of ink and processing necessary to form conductive metallic features. The nature of how energy is imparted to the deposit during processing is of particular relevance to additive manufacturing and potential mass production, as it dictates both the compatible materials that can be used and the speed with which the layer can be deposited. Use of processing techniques that enable the rapid processing of printed deposits, in conjunction with compatibility with materials typically used in additive manufacturing are discussed.

\section{References}

1. ASTM F2792-12a, Standard terminology for additive manufacturing technologies, ASTM International, West Conshohocken, PA, 2012.

2. J.H.P. Pallari, K.W. Dalgarno, J. Woodburn, Mass customization of foot orthoses for rheumatoid arthritis using selective laser sintering, IEEE Trans. Biomed. Eng. 57 (2010) 1750-1756.

3. L.E. Murr, S.M. Gaytan, F. Medina, H. Lopez, E. Martinez, B.I. Machade, D.H. Hernandez, L. Martinez, M.I. Lopez, R.B. Wicker, J. Bracke, Next-generation biomedical implants using additive manufacturing of complex, cellular and functional mesh arrays, J. Phil. Trans. Royal. Soc. A 368 (2010) 1999-2032.

4. M.D. Symes, P.J. Kitson, J. Yan, C.J. Richmond, G.J.T. Cooper, R.W. Bowman, T. Vilbrandt, L. Cronin, Integrated 3D-printed reactionware for chemical synthesis and analysis, Nature Chem. 4 (2012) 349-354.

5. M. Miodownik, Robotic craft: rapid-prototype technology may take the labor out of craft, but it also allows individually styled items to compete with those that have been mass-produced, Mater. Today 9 (2006) 6.

6. B.T. Wittbrodt, A.G. Glover, J. Laureto, G.C. Anzalone, D. Oppliger, J.L. Irwin, J.M. Pearce, Life-cycle economic analysis of distributed manufacturing with open-source 3-D printers, Mechatronics 23 (2013) 713-726.

7. I. Campbell, D. Bourell, I. Gibson, Additive manufacturing: rapid prototyping comes of age, Rapid Prototyping J. 18 (2012) $255-258$

8. D.D. Gu, W. Meiners, K. Wissenbach, R. Poprawe, Laser additive manufacturing of metallic components: materials, processes and mechanisms, Int. Mater. Rev. 57 (2012) 133-164.

9. K.V. Wong, A. Hernandez, A review of additive manufacturing, ISRN Mech. Eng. 2012 (2012) 208760.

10. N. Guo, M.C. Leu, Additive manufacturing: technology, applications and research needs, Frontiers Mech. Eng. 8 (2013) 215-243.

11. H. Devaraj, J. Travas-Sejdic, R. Sharma, N. Aydemir, D. Williams, E. Haemmerle, K.C. Aw, Bio-inspired flow sensor from printed PEDOT:PSS micro-hairs, Bioinspir. Biomim. 10 (2015) 016017.

12. G.M. Gratson, M. Xu, J.A. Lewis, Microperiodic structures: direct writing of three-dimensional webs, Nature 428 (2004) 386.

13. S. Wu, J. Serbin, M. Gu, Two-photon polymerisation for threedimensional micro-fabrication, J. Photochem. Photobiol. A: Chem. 181 (2006) 1-11. 
14. C. Sun, N. Fang, D.M. Wu, X. Zhang, Projection microstereolithography using digital micro-mirror dynamic mask, Sens. Actuators A: Phys. 121 (2005) 113-120.

15. N. Hopkinson, P. Dickens, Analysis of rapid manufacturing using layer manufacturing processes for production, P. I. Mech. Eng. C - J. MEC 217 (2003) 31-39.

16. C.E. Majewski, D. Oduye, H.R. Thomas, N. Hopkinson, Effect of infra-red power level on the sintering behaviour in the high speed sintering process, Rapid Prototyping J. 14 (2008) 155-160.

17. J.R. Tumbleston, D. Shirvanyants, N. Ermoshkin, R. Janusziewicz, A.R. Johnson, D. Kelly, K. Chen, R. Pinschmidt, J.P. Rolland, A. Ermoshkin, E.T. Samulski, J.M. DeSimone, Continuous liquid interface production of 3D objects, Science 347 (2015) 1349-1352.

18. X. Lu, S. Yang, J.R.G. Evans, Ultrasound-assisted microfeeding of fine powders, Particuology 6 (2008) 2-8.

19. A.J. Lopes, E. McDonald, R.B. Wicker, Integrating stereolithography and direct print technology for 3D structural electronics fabrication, Rapid Prototyping J. 18 (2012) 129-143.

20. S.H. Jang, S.T. Oh, I.H. Lee, H.-C. Kim, H.Y. Cho, 3-dimensional circuit device fabrication process using stereolithography and direct writing, Int. J. Precis. Eng. Manuf. 16 (2015) 1361-1367.

21. S.J. Leigh, R.J. Bradley, C.P. Purssell, D.R. Bilson, D.A. Hutchins, A simple low-cost conductive material for 3D printing of electronic sensors, PLoS One 7 (2012) e49365.

22. M.S. Mannoor, Z. Jiang, T. James, Y.L. Kong, K.A. Malatesa, W.O. Soboyejo, N. Verma, D.H. Gracias, M.C. McAlpine, "3D printed bionic ears, Nano Lett. 13 (2013) 2634-2639.

23. I.T. Nassar, T.M. Weller, An electrically-small, 3-D cube antenna fabricated with additive manufacturing, PAWR 2013, Santa Clara, CA, 2013, pp. 91-93.

24. C. Shemelya, F. Cedillos, E. Aguilera, D. Espalin, D. Muse, R. Wicker, E. McDonald, IEEE Sensors 15 (2015) 1280-1286.

25. C. Kim, D. Espalin, A. Cuaron, M.A. Perez, M. Lee, E. McDonald, R.B. Wicker, Cooperative tool path planning for wire embedding on additively manufactured curved surfaces using robot kinematics, J. Mech. Robot. 7 (2015) 021003.

26. E. Tekin, P.J. Smith, U.S. Schubert, Inkjet printing as a deposition and patterning tool for polymers and inorganic particles, Soft Matter 4 (2008) 703.

27. N. Reis, C. Ainsley, B. Derby, Ink-jet delivery of particle suspensions by piezoelectric droplet ejectors, J. Appl. Phys. 97 (2005) 094903.

28. J. Ebert, E. Ozkol, A. Zeichner, K. Uibel, O. Weiss, U. Koops, R. Telle, H. Fischer, Direct inkjet printing of dental prostheses made of zirconia, J. Dent. Res. 88 (2009) 673-676.

29. E. Sachs, M. Cima, P. Williams, D. Brancazio, J. Corrie, Three dimensional printing: rapid tooling and prototypes directly from a CAD model, J. Eng. Ind. 114 (1992) 481-488.

30. B.M. Wu, S.W. Borland, R.A. Giordano, L.G. Cima, E.M. Sachs, M.J. Cima, Solid free-form fabrication of drug delivery devices, J. Control. Release 40 (1996) 77-87.

31. B. Derby, Printing and prototyping of tissues and scaffolds, Science 338 (2012) 921.

32. M. Medina-Sanchez, C. Martinez-Domingo, E. Ramon, A. Merkoci, An inkjet-printed field-effect transistor for label-free biosensing, Adv. Funct. Mater. 24 (2014) 6291-6302.

33. Y. Zhang, J. Stringer, R. Grainger, P.J. Smith, A. Hodzic, Fabrication of patterned thermoplastic microphases between composite plies by inkjet printing, J. Comp. Mater. 49 (2014) 1907-1913.

34. J. Perelaar, P.J. Smith, D. Mager, D. Soltman, S.K. Volkman, V. Subramanian, J.G. Korvink, U.S. Schubert, Printed electronics: the challenges involved in printing devices, interconnects, and contacts based on inorganic materials, J. Mater. Chem. 20 (2010) 8446-8453.

35. Y. Zhang, C. Tse, D. Rahoulamin, P.J. Smith, Scaffolds for tissue engineering produced by inkjet printing, Cent. Eur. J. Eng. 2 (2012) 323.

36. C.C.W. Tse, S.S. Ng, J. Stringer, S. MacNeil, J.W. Haycock, P.J. Smith, Utilising inkjet printed paraffin wax for cell patterning applications, Int. J. Bioprinting 2 (2016) 35-44.

37. T. Boland, T. Xu, B. Damon, X. Cui, Application of Inkjet Printing to Tissue Engineering, Biotechnol. J. 1 (2006) 910.

38. V. Sanchez-Romaguera, M.A. Ziai, D. Oyeka, S. Barbosa, J.S.R. Wheeler, J.C. Batchelor, E.A. Parker, S.G. Yeates, Towards inkjet-printed low cost passive UHF RFID skin mounted tattoo paper tags based on silver nanoparticle tags, J. Mater. Chem. C 1 (2013) 6395.

39. S.B. Fuller, E.J. Wilhelm, J.A. Jacobson, Ink-jet printed nanoparticle microelectromechanical systems, J. Microelectromech. Syst. 11 (2002) 54-60.

40. S.H. Eom, S. Senthilarasu, P. Uthirakumar, S.C. Yoon, J. Lim, C. Lee, H.S. Lim, J. Lee, S.-H. Lee, Polymer solar cells based on inkjet-printed PEDOT:PSS layer, Org. Electr. 10 (2009) 536.

41. H.P. Le, Progress and trends in ink-jet printing technology, J. Imaging Sci. Technol. 42 (1998) 49-62.

42. D.B. Bogy, F.E. Talke, Experimental and theoretical study of wave propogation phenomena in drop-on-demand ink jet devices, IBM J. Res. Dev. 29 (1984) 314-321.

43. A.B.M. Buanz, R. Telford, I.J. Scowen, S. Gaisford, Rapid preparation of pharmaceutical co-crystals with thermal ink-jet printing, Cryst. Eng. Comm. 15 (2013) 1031-1035.

44. P.J. Smith, D.-Y. Shin, J.E. Stringer, N. Reis, B. Derby, Direct ink-jet printing and low temperature conversion of conductive silver patterns, J. Mater. Sci. 41 (2006) 4153.

45. M. Grouchko, A. Kamyshny, S. Magdassi, Formation of airstable copper-silver core-shell nanoparticles for inkjet printing, J. Mater. Chem. 19 (2009) 3057.

46. S.D. Hoath, W.-K. Hsiao, S.J. Jung, G.D. Martin, I.M. Hutchings, Drop speeds from drop-on-demand ink-jet print heads, J. Imaging Sci. Technol. 57 (2013) 010503.

47. K.A.M. Seerden, N. Reis, J.R.G. Evans, P.S. Grant, J.W. Halloran, B. Derby, Ink-jet printing of wax-based alumina suspensions, J. Am. Ceram. Soc. 84 (2001) 2514-2520, DOI: $10.1111 / \mathrm{j} .1151-2916.2001 . t b 01045 . x$.

48. B.-J. de Gans, E. Kazancioglu, W. Meyer, U.S. Schubert, Ink-jet printing polymers and polymer libraries using micropipettes, Macromol. Rapid Commun. 25 (2004) 292-296, DOI: $10.1002 /$ marc. 200300148 .

49. D. Xu, V. Sanchez-Romaguera, S. Barbosa, W. Travis, J. de Wit, P. Swan, S.G. Yeates, Inkjet printing of polymer solutions and the role of chain entanglement, J. Mater. Chem. 17 (2007) 4902-4907.

50. S.D. Hoath, I.M. Hutchings, G.D. Martin, T.R. Tuladhar, M.R. Mackley, D. Vadillo, Links between ink rheology, drop-ondemand jet formation, and printability, J. Imaging Sci. Technol. 53 (2009) 041208.

51. D.J. Hayes, W.R. Cox, M.E. Grove, Micro-jet printing of polymers and solder for electronics manufacturing, J. Eletron. Manu. 8 (1998) 209-216. 
52. J.E. Fromm, Numerical calculation of the fluid dynamics of drop-on-demand jets, IBM J. Res. Dev. 28 (1984) 322-333.

53. D.B. van Dam, C. Le Clerc, Experimental study of the impact of an ink-jet printed droplet on a solid substrate, Phys. Fluids 16 (2004) 3403-3414.

54. Y. Zhang, J. Stringer, R. Grainger, P.J. Smith, A. Hodzic, Improvements in carbon fibre reinforced composites by inkjet printing of thermoplastic polymer patterns, Phys. Status Solidi RRL 8 (2014) 56-60, DOI: 10.1002/pssr.201308149.

55. L. Bergstrom, Rheological properties of A12O3-SiC whisker composite suspensions, J. Mat. Sci. 31 (1996) 5257-5270.

56. B. Derby, N. Reis, Inkjet printing of highly loaded particulate suspensions, MRS Bull. 28 (2003) 815-818.

57. A. Denneulin, J. Bras, F. Carcone, C. Neuman, A. Blayo, Impact of ink formulation on carbon nanotube network organization within inkjet printed conductive films, Carbon 49 (2011) 2603-2614.

58. S. Jeong, D. Kim, J. Moon, Ink-jet printed organic-inorganic hybrid dielectrics for organic thin-film transistors, J. Phys. Chem. C 112 (2008) 5245-5249.

59. A. Lee, K. Sudau, K.H. Ahn, S.J. Lee, N. Willenbacher, Optimization of experimental parameters to suppress nozzle clogging in inkjet printing, Ind. Eng. Chem. Res. 51 (2012) 13195-13204.

60. C.N. Hoth, S.A. Choulis, P. Schilinsky, C.J. Brabec, High photovoltaic performance of inkjet printed polymer: fullerene blends, Adv. Mater. 19 (2007) 3973-3978.

61. B. Derby, Inkjet printing of functional and structural materials: fluid property requirements, feature stability and resolution, Ann. Rev. Mater. Res. 40 (2010) 395-414.

62. J.-U. Park, M. Hardy, S.J. Kang, K. Barton, K. Adair, D.K. Mukhopadhyay, C.Y. Lee, M.S. Strano, A.G. Alleyne, J.G. Georgiadis, P.M. Ferriera, J.A. Rogers, High-resolution electrohydrodynamic jet printing, Nature Mat. 6 (2007) 782-789.

63. K. Murata, J. Matsumoto, A. Tezuka, Y. Matsuba, H. Yokoyama, Super-fine inkjet printing: toward the minimal manufacturing system, Microsyst. Technol. 12 (2005) 2-7.

64. K. Takano, T. Kawabata, C.-F. Hsieh, K. Akiyama, F. Miyamaru, Y. Abe, Y. Tokuda, R.-P. Pan, C.-L. Pan, M. Hangyo, Fabrication of terahertz planar metamaterials using a superfine inkjet printer, App. Phys. Express 3 (2010) 016701.

65. C.C. Ho, K. Murata, D.A. Steingart, J.W. Evans, P.K. Wright, A super ink jet printed zinc-silver 3D microbattery, J. Micromech. Microeng. 19 (2009) 094013.

66. K. Murata, Direct fabrication of super-fine wiring and bumping by using inkjet process, Polytronic 2007, Odaiba, Tokyo, pp. 293-296, 2007.

67. H. Sirringhaus, T. Kawase, R.H. Friend, T. Shimoda, M. Inbasekaran, W. Wu, E.P. Woo, High resolution inkjet printing of all-polymer transistor circuits, Science 15 (2000) 2123-2126.

68. A.M.J. van den Berg, A.W.M. de Laat, P.J. Smith, J. Perelaar, U.S. Schubert, Geometric control of inkjet printed features using a gelating polymer, J. Mater. Chem. 17 (2007) 677-683.

69. M. Di Biase, R.E. Saunders, N. Tirelli, B. Derby, Inkjet printing and cell seeding thermoreversible photocurable gel structures, Soft Matter 7 (2011) 2639-2646.

70. K.F. Teng, R.W. Vest, Liquid ink jet printing with MOD inks for hybrid microcircuits, IEEE Trans. Components Hybrids Manuf. Technol. 11 (1988) 291.
71. S. Gamerith, A. Klug, H. Schreiber, U. Scherf, E. Moderegger, E.J.W. List, Direct ink-jet printing of $\mathrm{Ag}-\mathrm{Cu}$ nanoparticle and Ag-precursor based electrodes for OFET applications, Adv. Funct. Mater. 17 (2007) 3111.

72. B. Lee, Y. Kim, S. Yang, I. Jeong, J. Moon, A low-curetemperature copper nano ink for highly conductive printed electrodes, Curr. Appl. Phys. 9 (2009) e157.

73. S.H. Ko, H. Pan, C.P. Grigoropoulos, C.K. Luscombe, J.M.J. Frechet, D. Poulikakos, All-inkjet-printed flexible electronics fabrication on a polymer substrate by low-temperature highresolution selective laser sintering of metal nanoparticles, Nanotechnology 18 (2007) 345202.

74. H.J. Hwang, W.H. Chung, H.S. Kim, In situ monitoring of flash-light sintering of copper nanoparticle ink for printed electronics, Nanotechnology 23 (2012) 485205.

75. S.-H. Park, H.-S. Kim, Flash light sintering of nickel nanoparticles for printed electronics, Thin Solid Films 550 (2014) 575-581.

76. J.J.P. Valeton, K. Hermans, C.W.M. Bastiaansen, D.J. Broer, J. Perelaar, U.S. Schubert, G.P. Crawford, P.J. Smith, Room temperature preparation of conductive silver features using spin-coating and inkjet printing, J. Mater. Chem. 20 (2010) 543-546.

77. S.M. Bidoki, D.M. Lewis, M. Clark, A. Vakorov, P.A. Millner, D. McGorman, Ink-jet fabrication of electronic components, J. Micromech. Microeng. 17 (2007) 967.

78. Z.-K. Kao, Y.-H. Hung, Y.-C. Liao, Formation of conductive silver films via inkjet reaction system, J. Mater. Chem. 21 (2011) 18799-18803.

79. Y. Tang, W. He, G. Zhou, S. Wang, X. Yang, Z. Tao, J. Zhou, A new approach causing the patterns fabricated by silver nanoparticles to be conductive without sintering, Nanotechnology 23 (2012) 355304.

80. M. Layani, M. Grouchko, S. Shemesh, S. Magdassi, Conductive patterns on plastic substrates by sequential inkjet printing of silver nanoparticles and electrolyte sintering solutions, J. Mater. Chem. 22 (2012) 14349-14352.

81. D.-Y. Shin, G.-R. Yi, D. Lee, J. Park, Y.-B. Lee, I. Hwang, S. Chun, Rapid two-step metallization through physicochemical conversion of $\mathrm{Ag} 2 \mathrm{O}$ for printed "black" transparent conductive films, Nanoscale 5 (2013) 5043-5052.

82. A.L. Yarin, Drop impact dynamics: splashing, spreading, receding, bouncing, Ann. Rev. Fluid Mech. 38 (2006) 159-192.

83. A.M. Worthington, On the forms assumed by drops of liquids falling vertically on a horizontal plate, Proc. Roy. Soc. 25 (1876) 261-272.

84. I.V. Roisman, R. Rioboo, C. Tropea, Normal impact of a liquid drop on a dry surface: model for spreading and receding, Proc. Roy. Soc. A 458 (2002) 1411-1430.

85. C. Clanet, C. Beguin, D. Richard, D. Quere, Maximal deformation of an impacting drop, J. Fluid Mech. 517 (2004) 199-208.

86. Y. Son, C. Kim, D.H. Yang, D.J. Ahn, Spreading of an inkjet droplet on a solid surface with a controlled contact angle at low weber and reynolds numbers, Langmuir 24 (2008) 2900-2907.

87. S. Jung, I.M. Hutchings, The impact and spreading of a small liquid drop on a non-porous substrate over an extended time scale, Soft Matter 8 (2012) 2686-2696.

88. P.C. Duineveld, The stability of ink-jet printed lines of liquid with zero receding contact angle on a homogeneous substrate, J. Fluid Mech. 477 (2003) 175-200. 
89. J. Stringer, B. Derby, Limits to feature size and resolution in ink jet printing, J. Eur. Ceram. Soc. 29 (2009) 913-918.

90. S. Schiaffino, A.A. Sonin, Molten droplet deposition and solidification at low weber numbers, Phys. Fluids 9 (1997) 3172-3187.

91. T. Lim, S. Han, J. Chung, J.T. Chung, S. Ko, C.P. Grigoropoulos, Experimental study on spreading and evaporationof inkjet printed pico-liter droplet on a heated substrate, Int. J. Heat Mass Transfer 52 (2009) 431-441.

92. J. Kwon, S. Hong, H. Lee, J. Yeo, S.S. Lee, S.H. Ko, Direct selective growth of $\mathrm{ZnO}$ nanowire arrays from inkjet printed zinc acetate precursor on a heated substrate, Nano. Res. Lett. 8 (2013) 489.

93. D. Soltman, B. Smith, S.J.S. Morris, V. Subramanian, Inkjet printing of precisely defined features using contact-angle hysteresis, J. Colloid Int. Sci. 400 (2013) 135-139.

94. D. Bonn, J. Eggers, J. Indeku, J. Meunier, E. Rolley, Wetting and spreading, Rev. Mod. Phys. 81 (2009) 731-805.

95. R.D. Deegan, O. Bakajin, T.F. Dupont, G. Huber, S.R. Nagel, T.A. Witten, Capillary flow as the cause of ring stains from dried liquid drops, Nature 389 (1997) 827-829.

96. R.D. Deegan, O. Bakajin, T.F. Dupont, G. Huber, S.R. Nagel, T.A. Witten, Contact line deposits in an evaporating drop, Phys. Rev. E 62 (2000) 756-765.

97. J. Perelaer, P.J. Smith, C.E. Hendriks, A.M.J. van den Berg, U.S. Schubert, The preferential deposition of silica micro-particles at the boundary of inkjet printed droplets, Soft Matter 4 (2008) 1072-1078.

98. K.A. Baldwin, M. Granjard, D.I. Wilmer, K. Sefiane, D.J. Fairhurst, Drying and deposition of poly(ethylene oxide) droplets determined by Peclet number, Soft Matter 7 (2011) 7819-7826.

99. H. Hu, R.G. Larson, Marangoni effect reverses coffee-ring depositions, J. Phys Chem. B 110 (2006) 7090-7094.

100. D. Soltman, V. Subramanian, Inkjet-printed line morphologiesand temperature control of the coffee ring effect, Langmuir 24 (2008) 2224-2231.

101. R. Dou, B. Derby, Formation of coffee stains on porous surfaces, Langmuir 28 (2012) 5331-5338.

102. D.V. Ta, A. Dunn, T.J. Wasley, J. Li, R.W. Kay, J. Stringer, P.J. Smith, E. Esenturk, C. Connaughton, J.D. Shephard, Laser textured superhydrophobic surfaces and their applications for homogeneous spot deposition, Appl. Surf. Sci. 365 (2016) 153-159.

103. R. Bhardwaj, X. Fang, P. Somasundaran, D. Attinger, Selfassembly of colloidal particles from evaporating droplets: role of DLVO interactions and proposition of a phase diagram, Langmuir 26 (2010) 7833-7842.

104. B.J. de Gans, U.S. Schubert, Inkjet printing of well-defined polymer dots and arrays, Langmuir 20 (2004) 7789-7793.

105. R. Dou, T. Wang, Y. Guo, B. Derby, Ink-jet printing of zirconia: coffee staining and line stability, J. Am. Ceram. Soc. 94 (2011) 3787-3792.

106. J. Stringer, B. Derby, Formation and stability of lines produced by inkjet printing, Langmuir 26 (2010) 10365-10372.

107. T. Wang, M.A. Roberts, I.A. Kinloch, B. Derby, Inkjet printed carbon nanotube networks: the influence of drop spacing and drying on electrical properties, J. Phys. D 45 (2012) 315304.

108. E. Tekin, B.-J. de Gans, U.S. Schubert, Ink-jet printing of polymers - from single dots to thin film libraries, J. Mater. Chem. 14 (2004) 2627-2632.
109. D. Soltman, B. Smith, H. Kang, S.J.S. Morris, V. Subramanian, Methodology for inkjet printing of partially wetting films, Langmuir 26 (2010) 15686-15693.

110. J.-L. Lin, Z.-K. Kao, Y.-C. Liao, Preserving precision of inkjet-printed features with solvents of different volatilities, Langmuir 29 (2013) 11330-11336.

111. Y.V. Kalinin, V. Berejnov, R.E. Thorne, Contact line pinning by microfabricated patterns: effects of microscale topography, Langmuir 25 (2009) 5391-5397.

112. H.Y. Park, B.J. Kang, D. Lee, J.H. Oh, Control of surface wettability for inkjet printing by combining hydrophobic coating and plasma treatment, Thin Solid Films 526 (2013) $162-166$.

113. D.V. Ta, A. Dunn, T.J. Wasley, R.W. Kay, J. Stringer, P.J. Smith, C. Connaughton, J.D. Shephard, Nanosecond laser textured superhydrophobic metallic surfaces and their chemical sensing applications, Appl. Surf. Sci. 357 (2015) 248-254.

114. D.V. Ta, A. Dunn, T.J. Wasley, J. Li, R.W. Kay, J. Stringer, P.J. Smith, E. Esenturk, C. Connaughton, J.D. Shephard, Laser textured surface gradients, Appl. Surf. Sci. 371 (2016) 583-589.

115. C.E. Hendriks, P.J. Smith, J. Perelaer, A.M.J. ven den Berg, U.S. Schubert, Invisible' silver tracks produced by combining hot-embossing and inkjet printing, Adv. Funct. Mater. 18 (2008) 1031-1038.

116. Y. Yoshioka, G.E. Jabbour, Desktop inkjet printer as a tool to print conducting polymers, Synth. Metals 156 (2006) 779-783.

117. A. Morrin, O. Ngamna, E. O'Malley, N. Kent, S.E. Moulton, G.G. Wallace, M.R. Smyth, A.J. Killard, The fabrication and characterization of inkjet-printed polyaniline nanoparticle films, Electrochim. Acta 53 (2008) 5092-5099.

118. K. Kordas, T. Mustonen, G. Toth, H. Jantunen, M. Jantunen, C. Soldano, S. Talapatra, S. Kar, R. Vajtai, P.M. Ajayan, Inkjet printing of electrically conductive patterns of carbon nanotubes, Small 2 (2006) 1021-1025.

119. L. Huang, Y. Huang, J. Liang, X. Wan, Y. Chan, Graphenebased conducting inks for direct inkjet printing of flexible conductive patterns and their applications in electric circuits and chemical sensors, Nano Research 4 (2011) 675-684.

120. A.L. Dearden, P.J. Smith, D.-Y. Shin, N. Reis, B. Derby, P. O'Brien, A low curing temperature silver ink for use in ink-jet printing and subsequent production of conductive track, Macromol. Rapid Comm. 26 (2005) 315.

121. P. Buffat, J.-P. Borel, Size effect on the melting temperature of gold particles, Phys. Rev. A 13 (1976) 2287.

122. J. Stringer, B. Xu, B. Derby, Characterization of photoreduced silver organometallic salt deposited by inkjet printing, NIP2007, Anchorage, AK, pp. 960, 2007.

123. M.L. Allen, M. Aronniemi, T. Mattila, A. Alastalo, K. Ojanperä, M. Suhonen, H. Seppä, Electrical sintering of nanoparticle structures, Nanotechnology 19 (2008) 175201.

124. A.T. Alastalo, T. Mattila, M.L. Allen, M.J. Aronniemi, J.H. Leppäniemi, K.A. Ojanperä, M.P. Suhonen, H. Seppä, Rapid electircal sintering of nanoparticle structures, Mater. Res. Soc. Symp. Proc. 20091113 (2009) 1113-F02-07.

125. J. Perelaer, B.-J. de Gans, U.S. Schubert, Ink-jet printing and microwave sintering of conductive silver tracks, Adv. Mater. 18 (2006) 2101.

126. T. Shimoda, Y. Matsuki, M. Furusawa, T. Aoki, I. Yudasaka, H. Tanaka, H. Iwasawa, D. Wand, M. Miyasaka, Y. Takeuchi, 
Solution-processed silicon films and transistors, Nature 440 (2006) 783-786.

127. H. Klauk, Organic thin-film transistors, Chem. Soc. Rev. 39 (2010) 2643-2666.

128. C. Liao, F. Yan, Organic semiconductors in organic thin-film transistor-based chemical and biological sensors, Polym. Rev. 53 (2013) 352-406.

129. M.J. Małachowski, J. Żmija, Organic field-effect transistors, Opto-Electron. Rev. 18 (2010) 121-136.

130. V.C. Sundar, J. Zaumseil, V. Podzorov, E. Menard, R.L. Willett, T. Someya, M.E. Gershenson, J.A. Rogers, Elastomeric transistor stamps: reversible probing of charge transport in organic crystals, Science 303 (2004) 1644-1646.

131. O.D. Jurchescu, J. Baas, T.T.M. Palstra, Effect of impurities on the mobility of single crystal pentacene, Appl. Phys. Lett. 84 (2004) 3061-3063.

132. Y.-H. Kim, B. Yoo, J.E. Anthony, S.K. Park, Controlled deposition of a high-performance small-molecule organic single-crystal transistor array by direct ink-jet printing, Adv. Mater. 24 (2012) 497-502.
133. M.-B. Madec, P.J. Smith, A. Malandraki, N. Wang, J.G. Korvink, S.G. Yeates, Enhanced reproducibility of inkjet printed organic thin film transistors based on solution processable polymer-small molecule blends, J. Mater. Chem. 20 (2010) 9155-9160.

134. H. Minemawari, T. Yamada, H. Matsui, J. Tsutsumi, S. Haas, R. Chiba, R. Kumai, T. Hasegawa, Inkjet printing of singlecrystal films, Nature 475 (2011) 364-367.

135. H. Minemawari, T. Yamada, T. Hasegawa, Crystalline film growth of TIPS-pentacene by double-shot inkjet printing technique, Jpn. J. Appl. Phys. 53 (2014) 05HC10.

136. V. Sanchez-Romaguera, B.-M. Madec, S.G. Yeates, Inkjet printing of 3D metal-insulator-metal crossovers, React. Funct. Polym. 68 (2008) 1052-1058.

137. S.A. Algarni, T.M. Althagafi, P.J. Smith, M. Grell, An ionic liquid-gated polymer thin film transistor with exceptionally low “on" resistance, Appl. Phys. Lett. 104 (2014) 182107.

Cite this article as: Stringer J, Althagathi TM, Tse CC, Ta VD, Shephard JD, Esenturk E, Connaughton C, Wasley TJ, Li J, Kay RW \& Smith PJ: Integration of additive manufacturing and inkjet printed electronics: a potential route to parts with embedded multifunctionality. Manufacturing Rev. 2016, 3, 12. 